\title{
Analysis of DNA Adducts and Mutagenic Potency and Specificity in Rats Exposed to Acrylonitrile
}

Vernon E. Walker, $*, * *, \dagger, \downarrow, \S, \|$ Timothy R. Fennell, ${ }^{\dagger,}$ Dale M. Walker, $\|$,$\| Michael J. Bauer, { }^{\dagger \dagger}$

Patricia B. Upton, ${ }^{\dagger}$, George R. Douglas, ${ }^{\S \S}$ and James A. Swenberg**, $\dagger,+$

${ }^{\dagger}$ Chemical Industry Institute of Toxicology, Research Triangle Park, NC 27709, USA

*Wadsworth Center and ${ }^{\dagger}$ Bureau of Occupational Health and Injury Prevention, New York State Department of Health, Albany, New York 12201, USA

${ }^{\S}$ Department of Pathology and Laboratory Medicine, University of Vermont, Burlington, VT 05405, USA

\|The Burlington HC Research Group, Inc., Jericho, VT 05465, USA

${ }^{\perp}$ RTI International, Center for Bioorganic Chemistry, Research Triangle Park, NC 27709, USA

IExperimental Pathology Laboratories, Sterling VA 20167, USA

Hepartment of Environmental Sciences and Engineering, University of North Carolina at Chapel Hill, Chapel Hill, NC 27599, USA

${ }^{\S}$ Environmental Health Science and Research Bureau, Health Canada, Ottawa, Ontario K1A 0K9, Canada

*Corresponding Author: E-mail: vwalker@uvm.edu, Phone: 802-777-9394.

**These authors contributed equally to this work, each having designed and directed interfacing projects: Animal exposures and experiments for DNA adduct studies were conducted at the Chemical Industry Institute of Technology and animal exposures and experiments for Hprt gene mutation studies were performed at the New York State Department of Health. 
Table S1. Manifestation of Hprt Mutant T-cells in Spleen of Female F344 Rats Following 4 Weeks of Drinking Water Exposure to 0 or 500 ppm Acyrlonitrile ${ }^{a}$

\begin{tabular}{|c|c|c|c|}
\hline \multirow[b]{2}{*}{ Weeks post exposure } & \multicolumn{2}{|c|}{ Mutant Frequency $\times 10^{-6}$ (Animal number) } & \multirow[b]{2}{*}{$P$-value } \\
\hline & Controls $^{b}$ & Acrylonitrile-exposed & \\
\hline \multirow[t]{4}{*}{-4} & $2 / 6$ & & \\
\hline & 2.8 & & \\
\hline & 2.4 & & \\
\hline & Mean $\pm \mathrm{SE}=2.6 \pm 0.1$ & & \\
\hline \multirow[t]{6}{*}{0} & $3.32(\mathrm{ANC} 10)$ & $7.74(\mathrm{AN} 5 / 13)$ & \\
\hline & $2.93(\mathrm{ANC} 11)$ & $7.28(\mathrm{AN} 5 / 14)$ & \\
\hline & $2.93(\mathrm{ANC12})$ & $7.75(\mathrm{AN} 5 / 15)$ & \\
\hline & Mean $\pm \mathrm{SE}=3.0 \pm 0.2$ & $11.28(\mathrm{AN} 5 / 16)$ & \\
\hline & & $8.53(\mathrm{AN} 5 / 17)$ & \\
\hline & & Mean $\pm \mathrm{SE}=8.5 \pm 0.7$ & $<0.001 *$ \\
\hline \multirow[t]{6}{*}{2} & 3.15 (ANC13) & $9.94(\mathrm{AN} 5 / 18)$ & \\
\hline & $2.91(\mathrm{ANC} 14)$ & $9.22(\mathrm{AN} 5 / 19)$ & \\
\hline & $2.63(\mathrm{ANC} 15)$ & $10.12(\mathrm{AN} 5 / 20)$ & \\
\hline & Mean $\pm \mathrm{SE}=2.9 \pm 0.2$ & $8.57(\mathrm{AN} 5 / 21)$ & \\
\hline & & $11.25(\mathrm{AN} 5 / 22)$ & \\
\hline & & Mean $\pm \mathrm{SE}=9.8 \pm 0.5$ & $<0.001 *$ \\
\hline \multirow[t]{6}{*}{4} & 3.24 (ANC18) & $8.47(\mathrm{AN} 5 / 23)$ & \\
\hline & 2.64 (ANC19) & $9.06(\mathrm{AN} 5 / 24)$ & \\
\hline & 1.95 (ANC20) & $13.61(\mathrm{AN} 5 / 25)$ & \\
\hline & Mean $\pm \mathrm{SE}=2.6 \pm 0.4$ & $14.44(\mathrm{AN} 5 / 26)$ & \\
\hline & & $10.08(\mathrm{AN} 5 / 27)$ & \\
\hline & & Mean $\pm \mathrm{SE}=11.1 \pm 1.2$ & $<0.001 *$ \\
\hline \multirow[t]{5}{*}{6} & $2.23(\mathrm{ANC} 21)$ & $2.43(\mathrm{AN} 5 / 28)$ & \\
\hline & $2.00(\mathrm{ANC} 22)$ & $1.86(\mathrm{AN} 5 / 29)$ & \\
\hline & 1.52 (ANC23) & $2.12(\mathrm{AN} 5 / 31)$ & \\
\hline & Mean $\pm \mathrm{SE}=1.9 \pm 0.2$ & $2.74(\mathrm{AN} 5 / 32)$ & \\
\hline & & Mean $\pm \mathrm{SE}=2.3 \pm 0.2$ & 0.13 \\
\hline \multirow[t]{5}{*}{8} & $2.47(\mathrm{ANC} 24)$ & $8.20(\mathrm{AN} 5 / 33)$ & \\
\hline & 2.53 (ANC25) & 3.33 (AN5/34) & \\
\hline & 3.17 (ANC26) & $5.01(\mathrm{AN} 5 / 36)$ & \\
\hline & Mean $\pm \mathrm{SE}=2.7 \pm 0.2$ & $2.86(\mathrm{AN} 5 / 37)$ & \\
\hline & & Mean $\pm \mathrm{SE}=4.9 \pm 1.2$ & 0.09 \\
\hline
\end{tabular}

\footnotetext{
${ }^{a}$ Groups of rats were exposed to 0 or 500 ppm acrylonitrile in drinking water for 4 weeks, and then necropsied at $0,2,4,6$, or 8 weeks after the cessation of exposure for isolation of splenic lymphocytes and measurement of Hprt mutant frequencies using a T-cell cloning assay.

${ }^{b}$ Mean for all controls $(n=18)=2.6 \pm 0.1(\mathrm{SE}) \times 10^{-6}$.

* Significantly different at $p<0.05$ for pairwise tests, Mann-Whitney U-statistic or Student's t-test.
} 
Table S2. Dose-Response for Hprt Mutant T-cells in Spleen of Female F344 rats Following Drinking Water Exposure to Acrylonitrile ${ }^{a}$

\begin{tabular}{|c|c|c|c|c|}
\hline $\begin{array}{l}\text { Exposure level } \\
(\mathrm{ppm})\end{array}$ & $\begin{array}{l}\text { Average daily dose } \\
(\mathrm{mg} / \mathrm{kg} \text { bw/day })^{b}\end{array}$ & $\begin{array}{l}\text { Observed mutant frequency } \times 10^{-6} \\
\text { (Animal number) }\end{array}$ & $\begin{array}{l}\text { Average induced mutant } \\
\text { frequency }\left(\times 10^{-6}\right)^{c}\end{array}$ & $P$-value \\
\hline \multirow[t]{4}{*}{0} & 0 & 3.24 (ANC18) & & \\
\hline & & 2.64 (ANC19) & & \\
\hline & & 1.95 (ANC20) & & \\
\hline & & Mean $\pm \mathrm{SE}=2.6 \pm 0.4$ & --- & \\
\hline \multirow[t]{6}{*}{33} & 8 & $2.52(\mathrm{AN} 33 / 1)$ & & \\
\hline & & $4.06(\mathrm{AN} 33 / 2)$ & & \\
\hline & & $3.56(\mathrm{AN} 33 / 3)$ & & \\
\hline & & $3.36(\mathrm{AN} 33 / 4)$ & & \\
\hline & & $3.55(\mathrm{AN} 33 / 5)$ & & \\
\hline & & Mean $\pm \mathrm{SE}=3.4 \pm 0.3$ & $0.8 \pm 0.3$ & 0.057 \\
\hline \multirow[t]{6}{*}{100} & 21 & $6.93(\mathrm{AN} 1 / 5)$ & & \\
\hline & & $4.64(\mathrm{AN} 1 / 6)$ & & \\
\hline & & $5.90(\mathrm{AN} 1 / 7)$ & & \\
\hline & & $4.92(\mathrm{AN} 1 / 8)$ & & \\
\hline & & $3.10(\mathrm{AN} 1 / 9)$ & & \\
\hline & & Mean $\pm \mathrm{SE}=5.1 \pm 0.6$ & $2.5 \pm 0.6$ & $0.036^{*}$ \\
\hline \multirow[t]{6}{*}{500} & 76 & 8.47 (AN5/23) & & \\
\hline & & $9.06(\mathrm{AN} 5 / 24)$ & & \\
\hline & & $13.61(\mathrm{AN} 5 / 25)$ & & \\
\hline & & $14.44(\mathrm{AN} 5 / 26)$ & & \\
\hline & & $10.08(\mathrm{AN} 5 / 27)$ & & \\
\hline & & Mean $\pm \mathrm{SE}=11.1 \pm 1.2^{d}$ & $8.5 \pm 1.2$ & $0.018 *$ \\
\hline
\end{tabular}

${ }^{a}$ Groups of rats (4-week-old) were exposed to $0,33,100$ or $500 \mathrm{ppm}$ acrylonitrile in drinking water for 4 weeks, and then necropsied 4 weeks after the cessation of exposure for isolation of splenic lymphocytes and measurement of Hprt mutant frequencies using a cloning assay.

${ }^{b}$ Water consumption and body weight data were used to estimate the maximal average daily dose of acrylonitrile as described in the Materials and Methods (see Figure 1).

${ }^{c}$ The average induced mutant frequency equals the average experimentally observed mutant frequency minus the average background mutant frequency in control rats.

${ }^{e}$ Significantly elevated at $500 \mathrm{ppm}$ acrylonitrile compared to mutant frequency values at 33 or $100 \mathrm{ppm}$ acrylonitrile ( $p$-value $<0.05$ ), Dunn's method.

* Significantly different at $P<0.05$ for pairwise tests, Mann-Whitney U-statistic or Student's $t$-test. 
Table S3. Base Alterations in Hprt Exon 3 of Splenic T-Cells from F344 Rats Exposed to Acrylonitrile ${ }^{a}$

\begin{tabular}{|c|c|c|c|c|c|c|c|c|c|}
\hline \multirow{2}{*}{$\begin{array}{l}\text { Base }^{b} \text { and } \\
\text { mutation }^{c}\end{array}$} & \multicolumn{8}{|c|}{ Animal Number } & \multirow[b]{2}{*}{ Total } \\
\hline & AN5/9 & AN5/10 & AN5/18 & AN5/19 & AN5/20 & AN5/21 & AN5/22 & AN5/23 & \\
\hline $135 \mathrm{G} \rightarrow \mathrm{T}$ & & & & & & & 1 & & 1 \\
\hline $137 \mathrm{C} \rightarrow \mathrm{T}$ & 2 & & & & & & & & 2 \\
\hline $140 \mathrm{~A} \rightarrow \mathrm{T}$ & & & & & & & & 1 & 1 \\
\hline $143 \mathrm{G} \rightarrow \mathrm{A}$ & & & & & & & 1 & & 1 \\
\hline $148 \mathrm{G} \rightarrow \mathrm{C}^{e}$ & & 2 & & & & & & & 2 \\
\hline $154 \mathrm{G} \rightarrow \mathrm{A}^{d, e}$ & & & 1 & & 1 & & & 2 & 4 \\
\hline $157 \mathrm{G} \rightarrow \mathrm{T}$ & 1 & & & & & & & & 1 \\
\hline $164 \mathrm{~A} \rightarrow \mathrm{T}^{d e, f}$ & & 1 & & & & & & & 1 \\
\hline $168 \mathrm{~A} \rightarrow \mathrm{G}$ & 1 & & & & & & & & 1 \\
\hline $173 \mathrm{G} \rightarrow \mathrm{A}^{g}$ & 1 & & & & & & & & 1 \\
\hline $179 \mathrm{~A} \rightarrow \mathrm{G}$ & & & & & & 1 & & & 1 \\
\hline $182 \mathrm{~A} \rightarrow \mathrm{T}$ & & & & & & 1 & & & 1 \\
\hline $204 \mathrm{G} \rightarrow \mathrm{C}$ & & & & & & 1 & & & 1 \\
\hline $205 \mathrm{~A} \rightarrow \mathrm{G}$ & & & & & & 1 & & & 1 \\
\hline $205 \mathrm{~A} \rightarrow \mathrm{T}^{e}$ & & & & & 1 & & & & 1 \\
\hline $206 \mathrm{~A} \rightarrow \mathrm{G}^{f}$ & & & 1 & & & & & & 1 \\
\hline $206 \mathrm{~A} \rightarrow \mathrm{C}$ & & & & 1 & & & & & 1 \\
\hline $206 \mathrm{~A} \rightarrow \mathrm{T}^{g}$ & & & & & & 1 & & & 1 \\
\hline $207 \mathrm{G} \rightarrow \mathrm{C}^{f}$ & 1 & & & & & & & & 1 \\
\hline $208 \mathrm{G} \rightarrow \mathrm{A}^{d, e, g}$ & & & & & & & 1 & & 1 \\
\hline $209 \mathrm{G} \rightarrow \mathrm{A}$ & & & & 2 & & & & & 2 \\
\hline $215 \mathrm{~A} \rightarrow \mathrm{C}^{d}$ & & & & 1 & & & 1 & & 2 \\
\hline $218 \mathrm{~A} \rightarrow \mathrm{T}$ & & & 1 & & & & & & 1 \\
\hline $221 \mathrm{~T} \rightarrow \mathrm{C}^{d, f, g}$ & & & & & & 1 & & & 1 \\
\hline $221 \mathrm{~T} \rightarrow \mathrm{G}$ & 1 & & & & & & & & 1 \\
\hline $223 \mathrm{~T} \rightarrow \mathrm{C}^{f, g}$ & 1 & & & & & 1 & & 2 & 4 \\
\hline $233 \mathrm{~T} \rightarrow \mathrm{C}$ & & & & 1 & & & & & 1 \\
\hline $252 \mathrm{G} \rightarrow \mathrm{A}$ & & & & & & 1 & & & 1 \\
\hline $140+\mathrm{T}^{d}$ & & & & & & & & 1 & 1 \\
\hline $145-146$ & & & & & & & & & \\
\hline $\begin{array}{l}\mathrm{CTT} \rightarrow \mathrm{TGT} \\
181-183\end{array}$ & & 1 & & & & & & & 1 \\
\hline $\mathrm{CAC} \rightarrow \mathrm{CTG}$ & & & & & & 1 & & & 1 \\
\hline $196+\mathrm{A}$ & & & & & 1 & & & & 1 \\
\hline $207-212+\mathrm{G}^{d, f}$ & & 1 & & & & 1 & & & 2 \\
\hline $207-212-\mathrm{G}^{e, f, g}$ & & & & & & & & 1 & 1 \\
\hline $219-\mathrm{G}^{g}$ & & & & & & 1 & & & 1 \\
\hline Total & 8 & 5 & 3 & 5 & 3 & 11 & 4 & 7 & 46 \\
\hline
\end{tabular}

${ }^{a}$ Splenic T-cells were collected for the Hprt cloning and selection assay at 2 or 4 weeks after cessation of drinking water exposure of female F344 rats for 4 weeks to 500 ppm acrylonitrile.

${ }^{b}$ Numbering of the rat Hprt gene according to ${ }^{51}$.

${ }^{c}$ The mutation in the non-transcribed strand is reported.

${ }^{d}$ The same mutation was found in F344 rats exposed to 1,3-butadiene (Table $8 \mathrm{in}^{43}$ ).

${ }^{e}$ The same mutation was found in F344 rats exposed to butadiene diepoxide (Table $10 \mathrm{in}^{43}$ ).

${ }^{f}$ The same mutation was found in $\mathrm{B} 6 \mathrm{C} 3 \mathrm{~F} 1$ mice exposed to 1,3-butadiene (Table $7 \mathrm{in}^{43}$ ).

${ }^{g}$ The same mutation was found in mice exposed to butadiene diepoxide (Table 9 in ${ }^{43}$ ). 


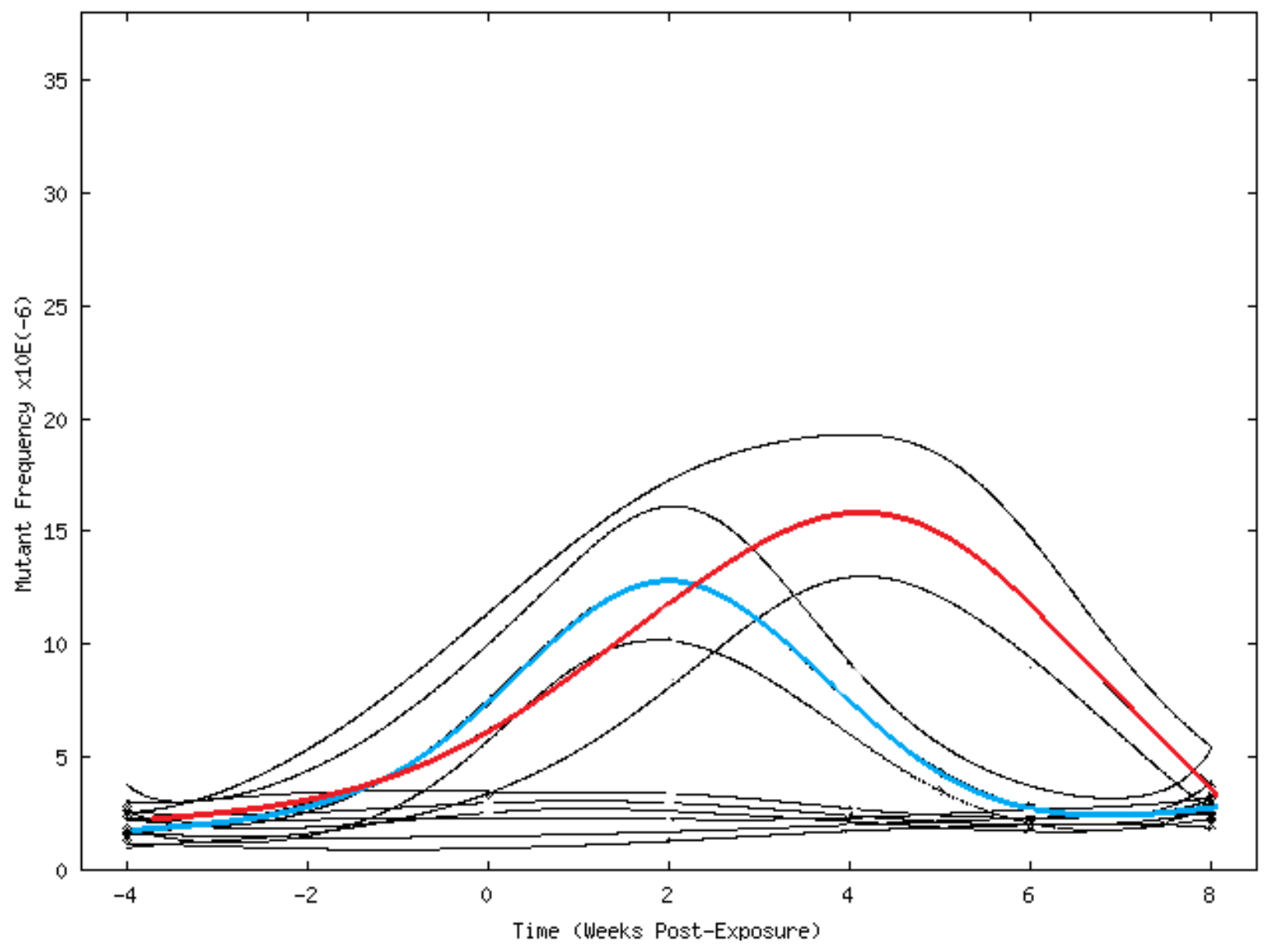

Figure S1. Relationships between the time elapsed since exposure and Hprt mutant frequencies in splenic T-cells of female F344 rats (4 weeks old) exposed for 4 weeks to 500 ppm acrylonitrile (blue curve) versus female B6C3F1 mice (4-5 weeks old) exposed for 4 weeks ( $6 \mathrm{~h} / \mathrm{day}, 5$ days/week) to 0 or 625 ppm 1,3-butadiene (red curve). Animals were necropsied before initiation of exposure (-4 week timepoint), after 2 weeks of exposure (-2 week timepoint), and up to 8 weeks after the last day of exposure Curves were fit to the Hprt mutant frequencies of individual rats exposed to acrylonitrile or mice exposed to 1,3-butadiene, 95\% confidence limits were determined (black curves), and the mutagenic potency estimate for each exposure scenario was obtained as described in the Materials and Methods. 